\title{
Knowledge, practices and concerns regarding exclusive breastfeeding for six months among mothers of infants in a suburban setting in Sri Lanka
}

\author{
B L Kamal Dhammika ${ }^{1}$, Nalika S Gunawardena ${ }^{2}$ \\ Sri Lanka Journal of Child Health, 2012; 41(1): 9-14
}

(Keywords: Exclusive breastfeeding; knowledge on breast feeding; opinions on breast feeding)

\begin{abstract}
Objective: To assess the knowledge, practices and concerns on six months exclusive breastfeeding among mothers.
\end{abstract}

Method: In a community based household survey in Kaduwela $\mathrm{MOH}$ area, 217 mothers with a baby aged $<1$ year were interviewed.

Results: Of the mothers, $45 \%$ had $>6$ months old babies. All mothers knew the recommended duration of exclusive breastfeeding. Advantages of breastfeeding to the child were well known. Among those with Ô month old babies, current exclusive breastfeeding rate was $85 \%$. Of those with $>6$ months old babies, $72 \%$ had exclusively breastfed them for 6 months. Mothers with ô months old babies not being exclusively breast fed and the mothers with $>6$ months old babies not exclusively breastfed for 6 months, indicated that having to return to work $(50 \%)$ and their opinions regarding amount of milk being inadequate $(44 \%)$ and nutrients being inadequate $(18 \%)$ as the reasons for not exclusively breast feeding up to the recommended age.

Conclusions: Current breast feeding rate and proportions exclusively breast fed were high. Knowledge regarding the duration of breast feeding and other essential aspects was good.

\section{Introduction}

The World Health Organization recommends that exclusive breastfeeding for the first 6 months is the optimum for feeding infants ${ }^{1}$. Breast milk is the best source of infant nutrition. It is easy to digest and

${ }_{1}$ Assistant Medical Practitioner, ${ }^{2}$ Senior Lecturer in
Community Medicine, Faculty of Medicine,
University of Colombo, Sri Lanka

(Received on 4 March 2011: Accepted on 29 April 2011) contains antibodies that can protect infants from bacterial and viral infections. Exclusive breastfeeding reduces infant mortality due to common childhood illnesses such as diarrhoea, pneumonia and recurrent respiratory tract infections and aids quicker recovery during illness. Breastfeeding is associated with lower rates of sudden infant death syndrome, childhood obesity, type 2 diabetes and leukaemia ${ }^{2}$. Therefore, exclusive breastfeeding is considered as a very important cost-effective public health intervention to reduce infant mortality in developing countries. The estimated reduction of infant mortality by promoting exclusive breastfeeding is $13 \%{ }^{3}$. Furthermore, exclusive breastfeeding contributes to the health and wellbeing of mothers, helps to space children, reduces the risk of ovarian and breast cancer, increases family and national resources, is a secure way of feeding and contributes to the protection of the natural environment ${ }^{4}$.

The Ministry of Health in Sri Lanka extended its policy on duration of exclusive breastfeeding, which was 4-6 months till 2005, to six months in response to $\mathrm{WHO}$ recommendation as a strategy to promote infant nutrition. In the Sri Lankan context breastfeeding a newborn has been a long honoured tradition. As the norm in Sri Lanka is to breastfeed exclusively, extending the period of exclusive breastfeeding was considered to be readily accepted. The most recent national data on breastfeeding status from Demographic and Health Survey (DHS) in 2006/2007 has indicated a sharp decline of proportions exclusively breastfed when disaggregated based on the age of the baby in months. Of the 0-1 month old infants $92 \%$ had been found to be exclusively breast fed while it dropped to $84 \%$ among 2-3 months old infants. It had further dropped to $53 \%$ among $4-5$ months old infants, and to $7.2 \%$ among 6-8 months old babies ${ }^{5}$. The overall proportion of exclusive breast feeding among 0-5 month old babies as per these data was $76 \%$. These findings indicate that the proportions of exclusive breast fed babies are rapidly decreasing as the child becomes older. The complementary feeding study of 
Sri Lanka in 2008 conducted in 22 districts using a triangulation of quantitative and qualitative methods found that $74 \%$ babies were exclusively breast fed only up to 4 months in spite of $85 \%$ mothers being aware that the period recommended for exclusive breast feeding is six months. At six months, exclusive breast feeding rate was only $9.9 \%$. The reasons given by mothers for not continuing exclusive breast feeding up to the recommended age was that they feel additional liquids should be fed when the baby is 4 or 5 months old. This and other anecdotal evidence indicate that mothers have concerns regarding achieving 6 months of exclusive breastfeeding.

\section{Objective}

The aim of this study was to assess knowledge, practices and concerns regarding breastfeeding exclusively for a period of six months, among mothers of infants.

\section{Method}

This was a community-based, descriptive, crosssectional study carried out in the Kaduwela Medical Officer of Health (MOH) area in the Colombo district. The period of data collection was February to May 2009. The study unit was defined as a mother with a baby less than or equal to one year at the time of the survey.

The sample size required was calculated based on estimates of practice of exclusive breast feeding among 4-5 month old infants ${ }^{5,7}$. The sample size required for the study was 217 . A cluster sampling technique with a Public Health Midwife (PHM) area defined as a cluster was used in selecting the study units. Seven PHM areas of the Kaduwela MOH area were randomly selected to be included in the study. A total of 31 eligible mothers were included from each PHM area. Identification of mothers was done using the Birth and Immunization Register available with the PHM. The rate of completeness of Birth and Immunization Register in Kaduwela $\mathrm{MOH}$ area was found to be very high (>95\%). The addresses of the mothers were also obtained from the same register. All the study units were visited at their homes by the principal investigator and a trained data collector who was not a member from the field health staff. Prior to the collection of data, the researcher obtained the informed verbal consent of the mothers by informing them of the purpose of the study. The survey was conducted during weekends and public holidays to facilitate inclusion of working mothers. A structured interviewer administered questionnaire was used to obtain data. The accepted methods to assess exclusive breastfeeding were adopted in the study. The questionnaire was pre-tested among a few mothers in the same area who were not included within the sample. Principal investigator and the trained data collector administered the questionnaire at the residence of the study unit. In the rare event when there was more than one baby less than or equal to one year, the information taken was related to the youngest baby. Care was taken not to disturb baby care or routine household work of the study unit and a location with minimal disturbance within the house/garden was selected for this purpose.

The collected data was tabulated using a spreadsheet and SPSS statistical software package was used for analysis. Ethical clearance was obtained from Ethics Review Committee of the Faculty of Medicine, University of Colombo.

\section{Results}

All who were invited for the study took part giving a response rate of $100 \%$. The study population was in the age range of 18 to 43 years, with a mean age of $29.7( \pm 5.3)$ years. The majority $(147,68 \%)$ of mothers in the study population belonged to the 2635 year age category.

Among study units 123 (57\%) had either passed Advanced Level or had achieved a higher education level. Study units were asked whether they were employed at the time of the survey even if they were on maternity or any other kind of leave. A total of $124(57 \%)$ was not employed and were housewives. Of the 98 employed mothers, approximately half (48, $52 \%)$ worked in the private sector. Others $(45,48 \%)$ were employed in the government sector. Only 4 of the employed study units were in occupations that required them to do night shifts.

Inquiring into living arrangements, a majority (146, $67 \%$ ) of the households of the study belonged to the extended family category as they lived together with parents of either the study unit or her husband. In a great majority $(209,96 \%)$ of the study units husbands were also living in the country and in the same household.

Approximately one third of husbands of the study units were self-employed $(84,39 \%)$ and another 81 (37\%) were private sector employees with only 52 $(24 \%)$ in the state sector.

A major proportion $(111,51 \%)$ of the study population had only one child. None has had twin babies. The youngest child in the majority of the 
study units $(119,55 \%)$ was Ò 6 months. Others (98, $45 \%$ ) had babies aged $>6$ months. Among the Oे 6 months old babies 48 (40\%) were <4 months old, 29 (24.5\%) were 4-5 months old, $32(27 \%)$ were 5-6 months old and $10(8.5 \%)$ were exactly 6 months old at the time of survey. The proportion of the male children of the study units $(117,54 \%)$ was higher compared to female children $(100,46 \%)$.

Knowledge of the mothers regarding breast feeding was assessed using a set of open and closed questions. All the mothers $(217,100 \%)$ in the study population knew that exclusive breast feeding is feeding babies only with breast-milk. All (217, $100 \%$ ) knew the recommendation of the duration of exclusive breastfeeding as complete 6 months. Most $(130,60 \%)$ also were aware that the recommended

duration of exclusive breastfeeding was recently increased by 2 months.

Assessing the knowledge regarding ideal time to initiate breastfeeding after the birth of the baby, 204 (94\%) of the study population were aware that ideally the newborn should be breastfed within one hour. A great majority $(189,87 \%)$ were also aware that babies should be breastfed on demand during the early neonatal period.

Two separate open questions were asked to assess the knowledge of the study units regarding advantages of exclusive breastfeeding to the child and to the mother and the frequency distribution of mothers who indicated correct responses are shown in Table 1 and Table 2.

Table 1

Distribution of the study population who gave correct responses regarding advantages of exclusive breastfeeding to the child

\begin{tabular}{|l|c|c|}
\hline \multicolumn{1}{|c|}{ Response } & Number (out of 217) & \% (out of 217) \\
\hline It improves the intelligence of the child & 214 & 98.6 \\
\hline It protects the child from common childhood illnesses & 216 & 99.5 \\
\hline It helps the child to gain weight appropriately & 211 & 97.2 \\
\hline It is a means of prevention of allergies, asthma and eczema in child & 183 & 84.3 \\
\hline
\end{tabular}

Table 2

Distribution of the study population who gave correct responses regarding advantages of exclusive breastfeeding to the mother

\begin{tabular}{|l|c|c|}
\hline \multicolumn{1}{|c|}{ Response } & Number (out of 217) & \% (out of 217) \\
\hline Its contribution to weight reduction to pre-pregnant weight & 140 & 64.5 \\
\hline Contraceptive effect & 93 & 42.9 \\
\hline Protection from breast cancer & 180 & 82.9 \\
\hline Protection from ovarian cancer & 86 & 39.6 \\
\hline Economical way of feeding & 210 & 96.8 \\
\hline
\end{tabular}

On requesting to name the sources of information on exclusive breastfeeding and about the recommended exclusive breast feeding duration as 6 months, the area PHM (213, 98\%) was mentioned by a great majority. Only $89(41 \%)$ indicated doctors (who attended to them during antenatal, natal, postnatal periods or who attended to baby) as a source of information on exclusive breast feeding.

Almost all the study units $(216,99.5 \%)$ had received information on feeding a child only with breast-milk up to 6 months during the antenatal period. All the study units $(217,100 \%)$ had been visited by the PHM in the early postnatal period and PHM had made inquiries/given advice about breast feeding during these visits.

The present study used accepted methods to assess exclusive breastfeeding rate at the time of the study by inquiring into the feeding history of the past 24 hours from the mothers of babies with Oे 6 months of age (Table 3 ). 
Table 3

Distribution of the study population with babies with $\leq 6$ months of age by whether they exclusively breastfed the child during the previous 24 hours

\begin{tabular}{|c|c|c|}
\hline Exclusively breastfed the child during the previous 24 hours & Frequency & Percentage \\
\hline Yes & 101 & 85 \\
\hline No & 18 & 15 \\
\hline Total & 119 & 100.0 \\
\hline
\end{tabular}

The exclusive breastfeeding rate was recorded as $85 \%$. Among the babies aged <4 months, the exclusive breastfeeding rate was $96 \%(46 / 48)$ while it was $77.5 \%$ (55/71) among 4 to Oे months old babies. Of the 18 babies who were not fed exclusively with breast milk during the past 24 hours, 3 were 3 months old, 2 were 4 months old, 9 were 5 months old and 4 were about to complete 6 months of age.

Study units with children Ò 6 months who were not exclusively breastfeeding during the past 24 hours, were asked about the fluids/foods they had introduced to their babies. All $(18,100 \%)$ had fed the babies with water. Other than water, they had fed fruit juices $(16,89 \%)$, formula milk $(9,52 \%)$, fruits (7, 37\%) and semi-solid rice/vegetables (7, 37\%). A great majority $(17,94.5 \%)$ had used a feeding bottle to feed the child with fluids. The 98 study units with babies $>6$ months of age were asked about the duration they exclusively breast fed the baby. A majority $(71,72.5 \%)$ of them had practised 6 months exclusive breastfeeding.

Mothers with Oे months old babies who were not being exclusively breast fed $(n=18)$ and the mothers with $>6$ months old babies who had not exclusively breastfed them for 6 months $(n=27)$ were asked about the reasons for not practising/for not having practised exclusive breastfeeding for 6 months. Not being entitled for maternity leave for 6 months $(23,50 \%)$ and their opinion that breast-milk was inadequate in amounts to fulfill the needs of the baby $(20,44.5 \%)$ and nutrients being inadequate to ensure growth of the baby $(17,38 \%)$ were the reasons given by them.

All the 93 employed mothers stated that not having full pay leave for 6 months was a reason for working mothers not to continue breast feeding up to the recommended period. A majority $(56,26 \%)$ of mothers were concerned about different opinions/ experiences expressed by their friends/relatives/ family members regarding breast feeding babies which they described as áonfusingô and not comparable to the messages from field health staff. Approximately half $(100,46.5 \%)$ of the mothers expressed their concern that breast feeding information was not stressed by the doctors who attended to the baby. They indicated the desire to get information on breast feeding and to clear their doubts from a professional source.

\section{Discussion}

As this was a community based study, it was possible to include all mothers with a baby less than or equal to one year, irrespective of whether they visited field clinics or not. Inclusion of only the mothers visiting the field clinics would have introduced a bias as the mothers who visit clinics are the ones that are more likely to have better exclusive breast feeding practices compared to those who do not visit clinics. The fact that the study was conducted during public holidays and Sundays ensured inclusion of both working and non-working mothers. Here again this avoided a bias which could have occurred if the study was conducted during weekdays which would have missed the working mothers as it is probable that non-working mothers are likely to have better exclusive breast feeding practices compared to working mothers.

Use of PHM as field investigators was purposely avoided as using them would have affected the accuracy of the information provided by the study units as PHM are the field health officers that advise them on breast feeding practices. Use of an interviewer administered questionnaire enabled the study to include eligible mothers of all education levels. Only the principal researcher and a trained data collector administered the questionnaire minimizing interviewer variations.

The question to assess the current exclusive breast feeding rates was based on recommended standard uniform question and enabling the findings directly comparable with other studies. In the present study, exclusive breast feeding rate was $85 \%$ among the infants Oे months age, which was estimated based on whether they gave only breast milk for the past 24 hours. This rate for babies aged <4 months and 4 to Ố months were $96 \%$ and $77.5 \%$ respectively. 
The rate of exclusive breast feeding even among the babies of 0-5 months in the DHS, the national study in $2006 / 2007$ was found to be lower $(75.6 \%)^{5}$ compared to the findings of the present study of exclusive breast feeding rate of $85 \%$ among the infants Oo months age. The complementary feeding study of Sri Lanka in 2008 found exclusive breast feeding rates as $74 \%, 45 \%$ and $10 \%$ at 4,5 and 6 months respectively. The numbers of babies assessed at each of these months were only 94, 56 and 10 at 4, 5 and 6 months respectively limiting the ability to make comparisons ${ }^{6}$. The studies done in previous years showed even less exclusive breast feeding rates. The study on exclusive breast feeding in Gampaha district in 2002 indicated that four months exclusive breastfeeding rate was $63.7 \%{ }^{8}$. The study in Beruwela $\mathrm{MOH}$ area, in 2006 on exclusive breast feeding showed that four month exclusive breast feeding was $61.6 \%$ and six months exclusive breastfeeding rate was $15.5 \%$ percent ${ }^{9}$. These lower figures in the past compared to the findings in the present study may be the results of continuous interventions carried out to improve breast feeding in the country by the Ministry of Health. The higher rate of exclusive breast feeding found in the present study in comparison to all above findings may also be attributed to Kaduwela being a semi-urban area and to the good field health services provided by PHM in the area as indicated in the results of the study.

Even in the light of having a high exclusive breast feeding rate, all efforts should be taken to improve it further considering the advantages of exclusive breast feeding to the baby and the mother. The present study described the reasons / concerns of mothers for not adhering to the full recommended period of exclusive breast feeding. The issue related to not being granted maternity leave for 6 months probably for the working mothers in private sector on temporary/contract basis can be corrected. Though the government policy on paid maternity leave also applies to the private sector its application to staff recruited on temporary/contract basis is often averted by way of temporarily discontinuing the services at the time of child birth. Mothers tend to rejoin the employment early as they are not receiving salaries for this period. The other related suggestion of the study units regarding 6 months maternity leave with full pay should receive attention of policy makers in the health sector.

Unfavourable opinions regarding insufficient breast milk and inadequacy of nutrients in breast milk, which were also reasons for not exclusively breast feeding are likely to have been generated through inadequate specific knowledge regarding these aspects even though when assessing knowledge of advantages of breast feeding to the baby many indicated that breast milk onelps the child to gain weight appropriatelyô This, taken together with preference of the study units to receive professional advice from doctors who attend to their babies over the advice from PHM, indicate that further improvement of exclusive breast feeding needs contribution of paediatricians and general practitioners.

\section{Conclusions and recommendations}

- The current rate of exclusive breastfeeding among mothers with babies < 6 months age and the proportion of mothers who had exclusively breast fed their babies among mothers with babies $>6$ months age were high. This can be taken as evidence of success of implementation of the breastfeeding policy within the study area within a short time period of four years.

- Knowledge regarding the duration of breast feeding and other essential aspects was good. Though many knew advantages of breast feeding to baby there were indications that certain key messages had not been addressed.

- The study recommends that first contact care doctors attending the infants, paediatricians and general practitioners should get actively involved in stressing the appropriateness and adequacy of only breast milk for babies till six months.

\section{References}

1. Butte NF, Lopez-Alarcon MG, Garza C. Nutrient adequacy of exclusive breastfeeding for the term infant during the first six months of life. Geneva: World Health Organization; 2002.

2. Ghai OP, Gupta P, Paul VK, editors. Ghai Essential Pediatrics, $6^{\text {th }}$ ed. New Delhi: Mehta Publishers; 2004.

3. Jones G, Steketee RW, Black RE, Bhutta ZA, Morris SS. How many child deaths can we prevent this year? Lancet 2003; 62: 65-71. http://dx.doi.org/10.1016/S01406736(03)13811$\underline{1}$

4. World Health Organization (2001), Exclusive Breastfeeding. Available from: http://www.who.int/nutrition/topics/exclusive br eastfeeding/en/index.html 
5. Department of Census and Statistics in collaboration with Ministry of Healthcare and Nutrition. Sri Lanka Demographic and Health Survey 2006/7. Department of Census and Statistics: May 2008.

6. Sri Lanka Complementary Feeding Study: Factors associated with complementary feeding in Sri Lanka. Ministry of Healthcare and Nutrition in collaboration with UNICEF. 2008:p66

7. Lawanga SK, Lemeshow S. Sample size determination in health studies. A Practical Manual. Geneva: World Health Organization, 1991.
8. Jayathilaka A, Fernando DA. Counseling on breast feeding: effectiveness of an educational intervention for health workers, in promoting breast feeding practices in the community. Journal of the College of Community Physicians of Sri Lanka 2004; 9: 28-31.

9. Agampodi SB, Agampodi TC, Kankanamge U, Piyaseeli D. Breasefeeding practices in a public health field practice area in Sri Lanka: a survival analysis. International Breastfeeding Journal 2007; 2:13

http://dx.doi.org/10.1186/1746-4358-2-13 